\title{
ULTRAMICROELETRODOS. PARTE II: CONSTRUÇÃo E APLICAÇÕES
}

S. M. Silva, C. R. Alves, A. N. Correia, R. M. Martins, A. L. R. Nobre ${ }^{\#}$, S. A. S. Machado, L. H. Mazo e L. A. Avaca* Instituto de Química de São Carlos - Universidade de São Paulo - CP 780 - 13560-970 - São Carlos - SP

Recebido em 16/10/96; aceito em 14/1/97

\begin{abstract}
ULTRAMICROELECTRODES. PART II: CONSTRUCTION AND APPLICATIONS. This work describes the techniques of construction and several applications of ultramicroelectrodes in electrochemistry and electroanalytical chemistry. Disc shaped UME are produced by embedding metal wires on insulating materials such as glass or epoxy resin. In the field of electrochemistry, UME have been applied in studies of the hydrogen evolution reaction and the electrocrystallization of metals. The negligible values of sensibility for ohmic drop and the enhanced mass transport rate by spherical diffusion are the main advantages of UME in these applications. New important conclusions regarding the phenomena under study were drawn from the experimental results. The applications in electroanalytical chemistry involved the determination of contaminants such as heavy metals and nitrites in natural waters and food products. The use of UME requires little sample manipulation and, in general, no need for oxygen removal or the addition of supporting electrolytes.
\end{abstract}

Keywords: ultramicroelectrodes; electroanalytical instrumentation; environmental analysis.

\section{INTRODUÇÃO}

A utilização de ultramicroeletrodos (UME) tem promovido uma expressiva ampliação dos limites experimentais em pesquisas nas mais diversas áreas da eletroquímica e, principalmente, da eletroanalítica. Devido às suas propriedades e características, este tipo de eletrodo tem se tornado uma ferramenta decisiva nas análises e determinações de: (a) contaminantes ambientais ${ }^{1,2}$, (b) moléculas bioquímicas, incluindo medicamentos ${ }^{3-6}$, (c) metais pesados ou substâncias tóxicas em alimentos ${ }^{7-9}$, etc. Além disto, vários processos eletródicos têm sido estudados sob uma nova ótica, originada da utilização dos UME. Neste caso, podese citar: (a) reações de desprendimento de gases ${ }^{10,11}$, (b) estudo de reações orgânicas ${ }^{12-15}$, (c) eletrocristalização ${ }^{16,17}$ e (d) estudos voltamétricos na ausência de eletrólito de suporte ${ }^{18,19}$.

Devido à freqüente utilização de UME nas diversas áreas como as descritas, vários artigos e revisões tratam de suas propriedades, vantagens e limitações ${ }^{20-24}$. Um artigo anterior deste Grupo foi publicado, analisando seus aspectos teóricos ${ }^{25}$.

Desta forma, os objetivos do presente trabalho são: (a) apresentar a metodologia empregada no Grupo de Materiais Eletroquímicos e Métodos Eletroanalíticos (GMEME) para a confecção de diversos tipos de UME, (b) discutir a instrumentação desenvolvida e (c) analisar as aplicações de UME nas diferentes linhas de pesquisas desenvolvidas no Grupo, tanto em eletroanálise como em eletroquímica fundamental.

\section{CONFECÇÃO E CARACTERIZAÇÃO DOS UME}

A maioria dos trabalhos nesta área desenvolvidos no Grupo envolvem UME na forma de microdiscos de $\mathrm{Pt}, \mathrm{Au}, \mathrm{Ni}, \mathrm{Co}$, Fe, etc. (Goodfellow Metals - USA), com diâmetros entre 10 e $50 \mu \mathrm{m}$, e de fibra de carbono com diâmetro de $7 \mu \mathrm{m}$ (CTA São José dos Campos).

\footnotetext{
${ }^{\#}$ Endereço permanente: Universidade Federal Rural do Rio de Janei-
} ro - Departamento de Química - Seropédica - RJ
Estes eletrodos são obtidos pelo embutimento dos microfios em tubos de vidro, polipropileno ou em resina epóxi (Reforplás S.A.). A junção do material eletródico com o contato externo (fio de cobre) é feita por tinta condutora de prata (Degussa S.A.) ou solda de ponto. $\mathrm{O}$ esquema de montagem é apresentado na figura 1 .

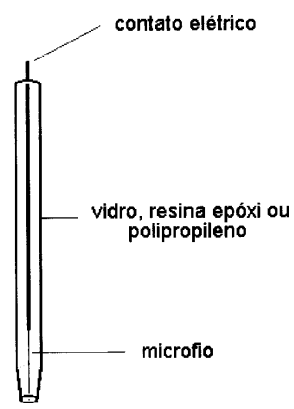

Figura 1. Representação esquemática de um UME na forma de disco, selado em vidro, polipropileno ou embutido em resina epóxi.

O procedimento de limpeza e pré-tratamento superficial é feito por polimento seqüencial com lixas de granulações $600,1500 \mathrm{e}$ 2000 até a exposição de um disco e alumina 0,25 e $0,01 \mu \mathrm{m}^{26}$, lavagem com ácido nítrico ${ }^{27}$ ou uma rápida lavagem com etanol $95 \%$, seguida de irradiação de ultra-som ${ }^{28}$. Wightman ${ }^{29}$ recomenda que seja evitado o polimento com pasta de diamante, pois correntes de estado estacionário irreprodutíveis foram obtidas sobre eletrodos de $\mathrm{Au}, \mathrm{Pt}$ e fibra de carbono, talvez pela dificuldade na remoção completa da pasta.

Devido à grande versatilidade dos UME, o GMEME vem empregando estes sistemas e desenvolvendo instrumentação adequada desde meados da década de 80. Estes estudos geraram um grande desenvolvimento na tecnologia de preparação e utilização deste tipo de eletrodo.

Anteriormente à sua aplicação em estudos eletroquímicos ou eletroanalíticos, os UME preparados devem ser testados quanto às suas propriedades específicas, visando detectar possíveis 
defeitos de fabricação, como infiltrações causadas pelo descolamento do fio metálico do material isolante.

Uma forma usual de caracterização de UME consiste na realização de voltametrias cíclicas em uma solução contendo um par redox de comportamento conhecido. Um dos sistemas usualmente escolhidos consiste em uma solução de $\mathrm{K}_{3} \mathrm{Fe}(\mathrm{CN})_{6}$ $1 \times 10^{-3} \mathrm{M}$ em $\mathrm{H}_{2} \mathrm{SO}_{4} \mathrm{pH} 2,3$. Um exemplo do voltamograma cíclico obtido para este sistema em um UME de fibra de carbono $(\phi=7 \mu \mathrm{m})$ é mostrado na figura $2 \mathrm{~A}$ a 0,01 e $0,1 \mathrm{~V} \mathrm{~s}^{-1}$. A forma sigmoidal da onda em estado estacionário, a $0,01 \mathrm{~V} \mathrm{~s}^{-1}$, assim como a ausência de histerese, reflete um comportamento característico de UME. No experimento a $0,1 \mathrm{~V} \mathrm{~s}^{-1}$, uma certa histerese é observada, com o início da definição do pico de corrente, característico de experimentos com eletrodos de dimensões convencionais, indicando que a velocidade de varredura começa a ser mais rápida que o transporte de massa para o eletrodo. Uma análise da relação $\mathrm{E} v s . \log \left(\mathrm{I}_{\mathrm{d}}-\mathrm{I}\right) / \mathrm{I}$ obtida da figura $2 \mathrm{~A}$ mostra a concordância do valor obtido da tangente com aquele esperado para uma reação reversível com transferência de um elétron $(0,060 \mathrm{~V} /$ déc), segundo a equação de Nernst. Esta análise é mostrada na figura 2B para ambas velocidades de varredura.

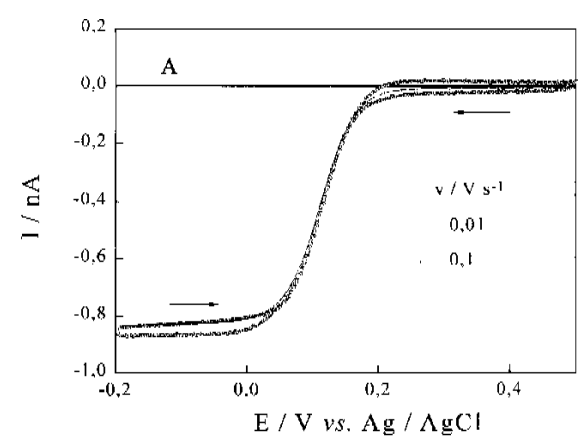

(A)

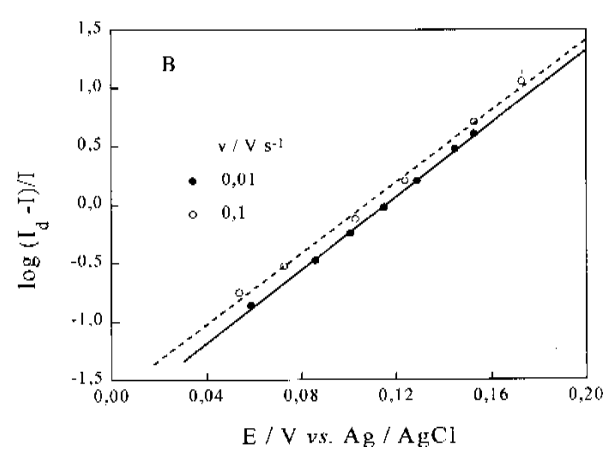

(B)

Figura 2. (A) Voltamogramas cíclicos de $\mathrm{K}_{3} \mathrm{Fe}(\mathrm{CN})_{6} 1 \times 10^{-3} \mathrm{M}+\mathrm{H}_{2} \mathrm{SO}_{4}$ $(\mathrm{pH}=2,3)$ deaerado, sobre UME de fibra de carbono $(\phi=7 \mu \mathrm{m})$ a 0,01 e $0,1 \mathrm{~V} \mathrm{~s}^{-1}, e(B)$ "log-plot" obtido dos voltamogramas anteriores.

Outra forma de caracterização superficial do UME, apresentada na figura 3 , consiste na observação do comportamento voltamétrico da superfície de metais em meios aquosos ácidos ou básicos. Como exemplo, tem-se UME de Pt $(\phi=40 \mu \mathrm{m}) \mathrm{e}$ de $\mathrm{Au}(\phi=25 \mu \mathrm{m})$ em $\mathrm{NaOH} 0,5 \mathrm{M}$ e $\mathrm{H}_{2} \mathrm{SO}_{4} 0,5 \mathrm{M}$, respectivamente, a $0,1 \mathrm{~V} \mathrm{~s}^{-1}$. Os perfis voltamétricos são concordantes com aqueles reportados na literatura ${ }^{30,31}$, indicando a ausência de infiltrações por trincas entre o metal e o vidro, ou resina, e as boas condições de pureza do eletrólito. As infiltrações causariam uma inclinação do perfil voltamétrico ao longo do eixo $\mathrm{x}$, devido à grande resistência do filme de eletrólito formado nas trincas. As impurezas presentes inibiriam os picos
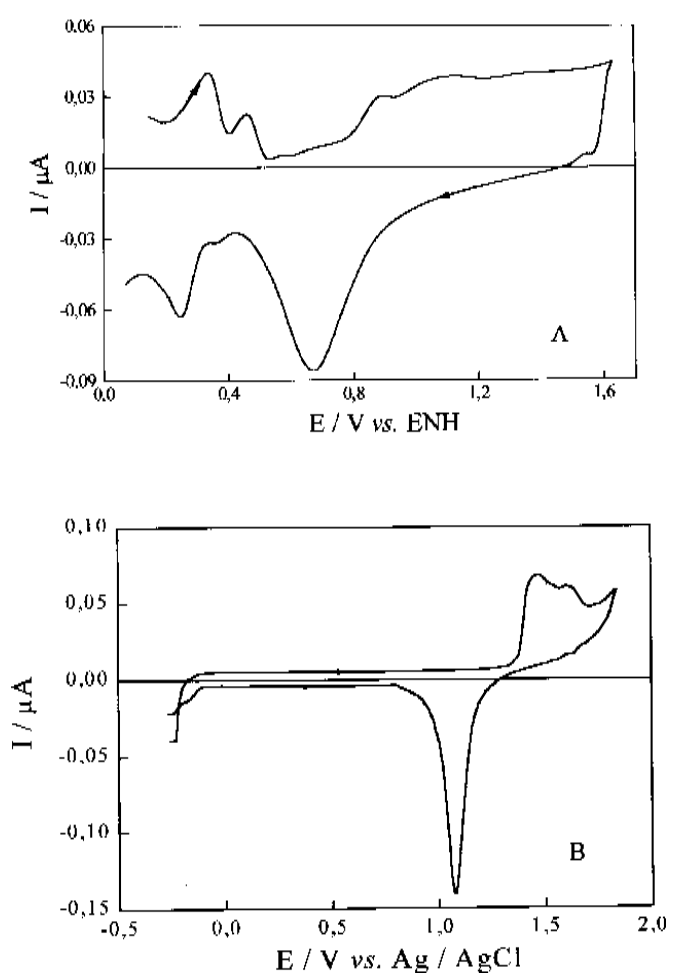

Figura 3. Voltamogramas cíclicos para UME de: (A) Pt $(\phi=40 \mu \mathrm{m})$ em $\mathrm{NaOH} 0,5 \mathrm{M}$, a $0,1 \mathrm{Vs}^{-1}$ e (B) $\mathrm{Au}(\phi=25 \mu \mathrm{m}) \mathrm{em}_{2} \mathrm{SO}_{4} 0,5 \mathrm{M}$ a $0,1 \mathrm{~V} \mathrm{~s}^{-1}$. Soluções deaeradas.

de adsorção/dessorção de hidrogênio ou de formação/redução dos óxidos sobre a superfície dos eletrodos.

Neste caso não se observam os perfis voltamétricos de formatos sigmoidais como aqueles do caso anterior pois as reações envolvidas são processos superficiais, sem qualquer controle difusional.

\section{INSTRUMENTAÇÃO}

Uma das limitações encontradas no uso de UME é a exigência de uma instrumentação adequada para medir correntes da ordem de nano a picoampères, sendo necessários amplificadores sensíveis a este nível de sinal. Isto implica na necessidade de se eliminar ruídos externos com a utilização de filtros de ruídos ou gaiolas de Faraday.

A instrumentação esquematizada na figura 4 foi desenvolvida no Grupo ${ }^{32}$, visando exclusivamente superar os problemas descritos acima na utilização de UME. Ela é composta por: a) interface controladora/conversora AD/DA de doze bits com 24 canais TTL usando um circuito integrado 8255. Esta placa foi construída para operar em microcomputadores tipo IBM-PC, na faixa de $\pm 10 \mathrm{~V}$, com uma velocidade de conversão nominal da ordem de $250 \mathrm{~ms}$ limitada pelo "software", $b$ ) picoamperímetro para utilização em sistemas de dois eletrodos, com fundo de escala de $200 \mathrm{pA}$ até $2 \mathrm{~mA}$ com saída de $1 \mathrm{~V}$ por fundo de escala e c) "software" AVOLM, escrito em Quick-Basic e Assembler, que permite a execução automática de várias técnicas eletroquímicas, tais como voltametria cíclica, voltametria de redissolução de corrente contínua, voltametria de redissolução de pulso diferencial, voltametria de onda quadrada, curvas de polarização e cronoamperometria. Este programa permite a geração de arquivos de dados tanto na forma binária como em ASCII. Com a utilização desta instrumentação, os experimentos com UME podem ser totalmente controlados por computador, com evidente ganho em reprodutibilidade, rapidez e facilidade no tratamento matemático dos dados. 


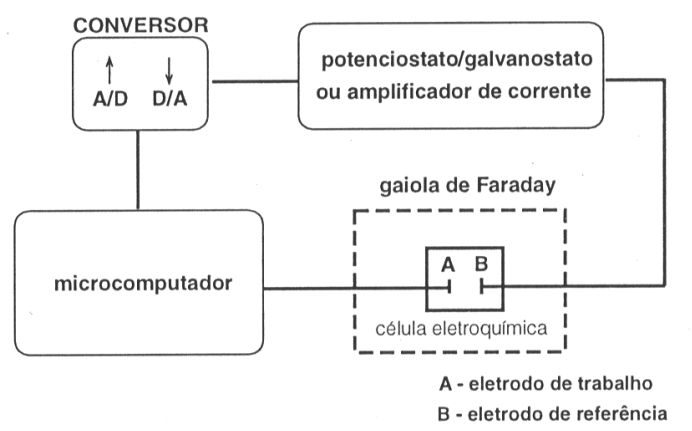

Figura 4. Diagrama da instrumentação eletroquímica desenvolvida para a utilização de UME.

\section{APLICAÇÕES EM ELETROQUÍMICA E ELETROANALÍTICA}

O GMEME tem desenvolvido várias linhas de pesquisas empregando UME de diversos materiais. A seguir será feita uma análise resumida de cada aplicação, com ênfase nas vantagens obtidas pela utilização dos UME, tanto na área da eletroquímica como, e principalmente, na área de eletroanalítica utilizando-se principalmente técnicas de varreduras de potencial (linear, de pulso e de onda quadrada), de redissolução anódica e de redissolução potenciométrica.

\section{A. Eletroquímica}

\section{i) Reação de desprendimento de hidrogênio sobre UME de $\mathrm{Pt}^{10}$}

A elucidação da cinética da reação de desprendimento de hidrogênio (RDH) está baseada na determinação de parâmetros como coeficiente angular de Tafel, densidade de corrente de troca e grau de recobrimento por $\mathrm{H}_{\mathrm{ads}}$, a partir de curvas de polarização de estado estacionário. Um dos problemas oriundos de reações que envolvem a geração de espécies gasosas relaciona-se com a grande queda ôhmica causada em parte pela formação da cortina de bolhas sobre a superfície eletródica. Neste caso, o uso de UME apresenta-se como uma alternativa apropriada, pois as correntes que circulam por tais sistemas são tão pequenas que possibilitam apenas a formação de quantidades mínimas de gases, que difundem rapidamente para a solução, sem nucleação de bolhas. Isto, juntamente com a dimensão das correntes aplicadas tornam a queda ôhmica do sistema praticamente desprezível.

$\mathrm{Na}$ figura 5 são apresentadas as curvas de polarização para a $\mathrm{RDH}$ sobre UME de $\mathrm{Pt}(\phi=25 \mu \mathrm{m})$ em meio de $\mathrm{H}_{2} \mathrm{SO}_{4} 0,5 \mathrm{M}$, com a temperatura variando entre 25 e $75^{\circ} \mathrm{C}$. Uma característica muito interessante desta família de curvas é a densidade de corrente máxima de aproximadamente $2 \mathrm{~A} \mathrm{~cm}^{-2}$. Apesar do alto valor da densidade de corrente, a corrente aplicada é apenas cerca de $10 \mu \mathrm{A}$, insuficiente para gerar bolhas de $\mathrm{H}_{2}$ na superfície do eletrodo. A análise das curvas de polarização por regressão nãolinear possibilitou estabelecer um mecanismo do tipo:

$\mathrm{Pt}+\mathrm{H}^{+}+\mathrm{e}^{-} \rightarrow \mathrm{PtH}_{\mathrm{ads}}$

(Etapa de Volmer)

$\mathrm{PtH}_{\mathrm{ads}}+\mathrm{H}^{+}+\mathrm{e}^{-} \rightarrow \mathrm{Pt}+\mathrm{H}_{2}$

(Etapa de Heyrovsky)

com a etapa de Heyrovsky sendo determinante da velocidade da reação.

A automação dos experimentos permitiu uma excelente reprodutibilidade dos dados experimentais, devido principalmente ao condicionamento da superfície, realizado antes da tomada de cada valor de potencial na curva de polarização.

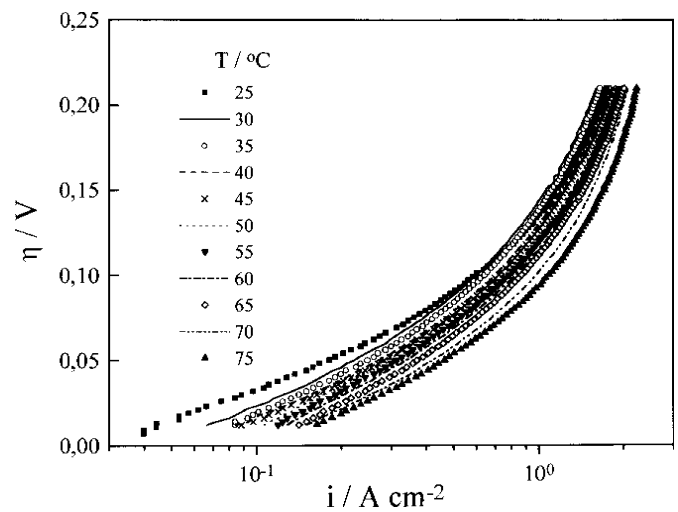

Figura 5. Curvas de polarização para a RDH sobre UME de Pt $(\phi=25 \mu \mathrm{m})$ em meio de $\mathrm{H}_{2} \mathrm{SO}_{4} 0,5 \mathrm{M}$, a diferentes temperaturas.

\section{ii) Reação de desprendimento de hidrogênio sobre UME de $\mathrm{Ni}$ e $\mathrm{Hg}^{33}$}

Na figura 6 tem-se as curvas de polarização para a RDH sobre: (A) Ni eletrodepositado sobre UME de $\mathrm{Pt}(\phi=40 \mu \mathrm{m})$, a partir de uma formulação modificada do banho de Watts $\left(\mathrm{NiSO}_{4} 330 \mathrm{~g} \mathrm{~L}^{-1}\right.$ $\left.+\mathrm{Ni}\left(\mathrm{NO}_{3}\right)_{2} 45 \mathrm{~g} \mathrm{~L}^{-1}+\mathrm{H}_{3} \mathrm{BO}_{3} 37 \mathrm{~g} \mathrm{~L}^{-1}\right)$, em NaOH 0,5 M e (B) $\mathrm{Hg}$ eletrodepositado sobre UME de $\mathrm{Pt}(\phi=40 \mu \mathrm{m})$, a partir de uma solução de $\mathrm{Hg}\left(\mathrm{NO}_{3}\right)_{2} \quad 1,26 \times 10^{-3} \mathrm{M}+\mathrm{KNO}_{3} 0,1 \mathrm{M}$, em meio de $\mathrm{HClO}_{4}$ 0,5 M, com a temperatura variando entre 25 e $70{ }^{\circ} \mathrm{C}$.

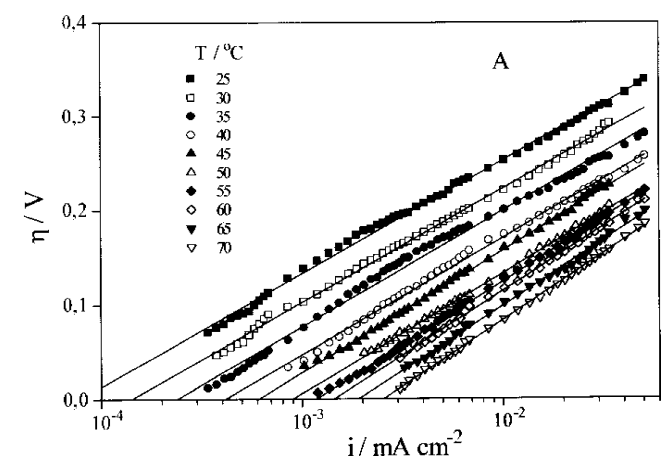

(A)

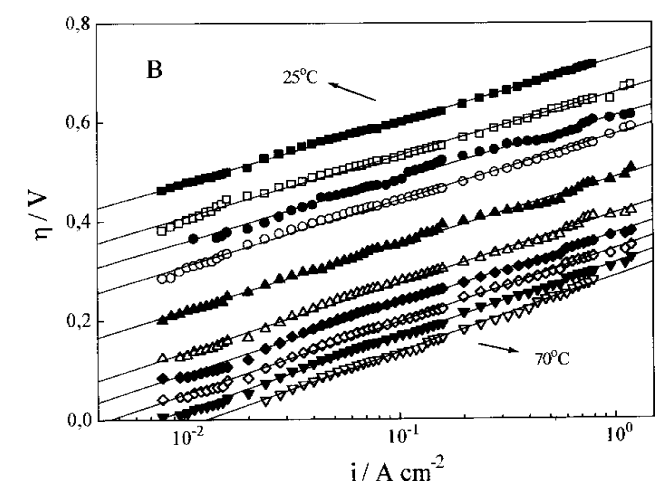

(B)

Figura 6. (A) Curvas de polarização para a RDH sobre $\mathrm{Ni}$ eletrodepositado sobre UME de Pt $(\phi=40 \mu \mathrm{m})$ em meio de $\mathrm{NaOH} 0,5$ $M$, a diferentes temperaturas e (B) Curvas de polarização para a $R D H$ sobre $\mathrm{Hg}$ eletrodepositado sobre UME de Pt $(\phi=40 \mu \mathrm{m})$ em meio de $\mathrm{HClO}_{4}$ O,5 $\mathrm{M}$, a diferentes temperaturas.

A partir dos valores das correntes de troca, $i_{0}$, para cada reta, determinou-se, pelo gráfico de Arrhenius, as energias de ativação 
para os dois processos eletródicos. Sobre $\mathrm{Ni}$, este valor foi de 64,6 $\mathrm{kJ} \mathrm{mol}^{-1}$, enquanto que sobre $\mathrm{Hg}$ foi de $179,3 \mathrm{~kJ} \mathrm{~mol}^{-1}$. Uma análise destas energias de ativação tornou possível associá-las aos seguintes complexos ativados: $\left(\mathrm{Ni}-\mathrm{H} \cdots \mathrm{H}^{\cdots} \mathrm{OH}\right)^{\#}$ e $\left(\mathrm{Hg} \cdot \mathrm{H}^{\cdots} \mathrm{H}_{2} \mathrm{O}^{+}\right)^{\#}$, respectivamente. Isto significa que a reação de desprendimento de hidrogênio sobre ambos metais ocorre por um mecanismo VolmerHeyrovsky. Entretanto, sobre Ni a etapa determinante da velocidade é a de Heyrovsky, sendo a de Volmer determinante sobre $\mathrm{Hg}$. Como no item anterior, a utilização de UME possibilitou um grande aumento na reprodutibilidade dos dados experimentais pela automação, bem como livrou estes valores da interferência da queda ôhmica do sistema.

\section{iii) Eletrocristalização ${ }^{34}$}

Os estágios iniciais de eletrocristalização geralmente estão associados a processos de nucleação bi ou tridimensionais, onde o número de núcleos formados depende fortemente do sobrepotencial, com formação instantânea ou progressiva, e seu crescimento ocorrendo com controle por transferência eletrônica ou de massa.

Foram feitos estudos de nucleação eletroquímica de $\mathrm{Cu}, \mathrm{Hg}$ e Ni sobre UME de Pt $(\phi=40 \mu \mathrm{m})$. Nas figuras 7, 8 e 9 tem-se os respectivos voltamogramas cíclicos e saltos potenciostáticos obtidos a partir de uma solução de $\mathrm{CuSO}_{4} 0,1 \mathrm{M}$ em $\mathrm{H}_{2} \mathrm{SO}_{4} 0,5 \mathrm{M}$; $\mathrm{Hg}\left(\mathrm{NO}_{3}\right)_{2}, 1,26 \times 10^{-3} \mathrm{M}$ em $\mathrm{KNO}_{3} 0,1 \mathrm{M}$ e de um banho de Watts modificado (composto de $\mathrm{NiSO}_{4} 330 \mathrm{~g} \mathrm{~L}^{-1}+\mathrm{Ni}\left(\mathrm{NO}_{3}\right)_{2} 45 \mathrm{~g} \mathrm{~L}^{-1}+$ $\left.\mathrm{H}_{3} \mathrm{BO}_{3} 37 \mathrm{~g} \mathrm{~L}^{-1}\right)$.
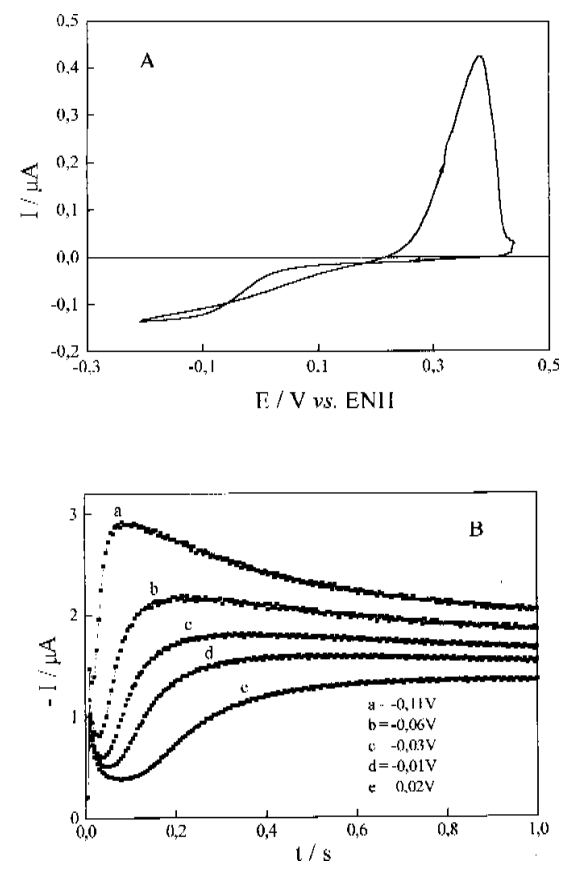

Figura 7. (A)Voltamograma cíclico para a deposição e redissolução de $\mathrm{Cu}$ sobre UME de Pt $(\phi=40 \mu \mathrm{m})$, em $\mathrm{CuSO}_{4} \mathrm{O}, 1 \mathrm{M}+\mathrm{H}_{2} \mathrm{SO}_{4} \mathrm{O}, 5$ $M, a 0,1 \mathrm{Vs}^{-1}$ e (B) Transientes potenciostáticos para a nucleação de Cu sobre UME de Pt $(\phi=40 \mu \mathrm{m})$ no mesmo eletrólito.

Em eletrodos de tamanho convencional, o modelo de Scharifker e Hills ${ }^{35}$ é o que melhor representa a maioria dos sistemas estudados. Entretanto, suas equações cinéticas não se adequaram ao comportamento obtido experimentalmente sobre UME, já que o modelo de Scharifker e Hills é válido somente para crescimento de núcleos controlados por difusão linear semiinfinita. Assim, desenvolveu-se um modelo considerando a difusão esférica para a superfície de um UME em forma de dis$\operatorname{co}^{21,36}$, cujo ajuste numérico ${ }^{37}$ indica nucleação instantânea para $\mathrm{Cu}$ e progressiva para $\mathrm{Hg}$. No caso do $\mathrm{Ni}$, cujo crescimento dos
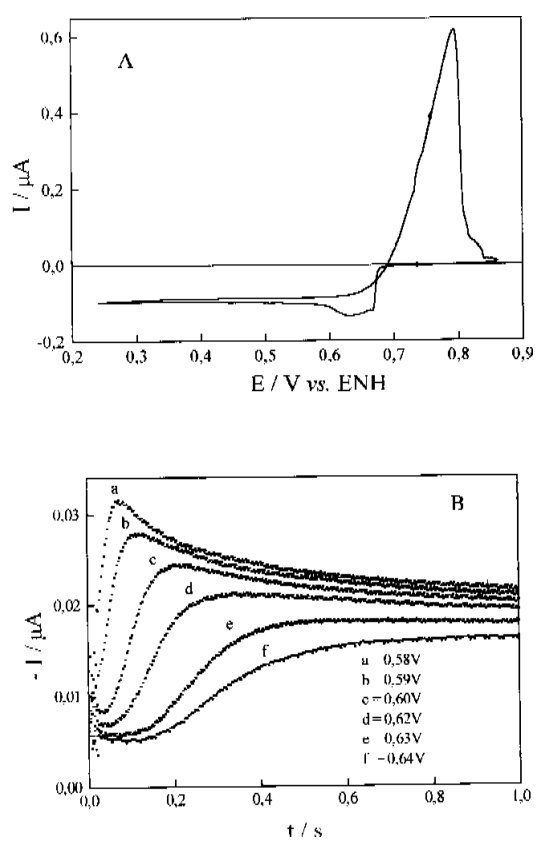

Figura 8. (A)Voltamograma cíclico para a deposição e redissolução de $\mathrm{Hg}$ sobre UME de Pt $(\phi=40 \mu \mathrm{m})$, em $\mathrm{Hg}\left(\mathrm{NO}_{3}\right)_{2} \quad 1,26 \times 10^{-3} \mathrm{M}+$ $\mathrm{KNO}_{3}$ 0,1 M, a $0,1 \mathrm{Vs}^{-1}$ e (B) Transientes potenciostáticos para a nucleação de $\mathrm{Hg}$ sobre UME de Pt $(\phi=40 \mu \mathrm{m})$ no mesmo eletrólito.
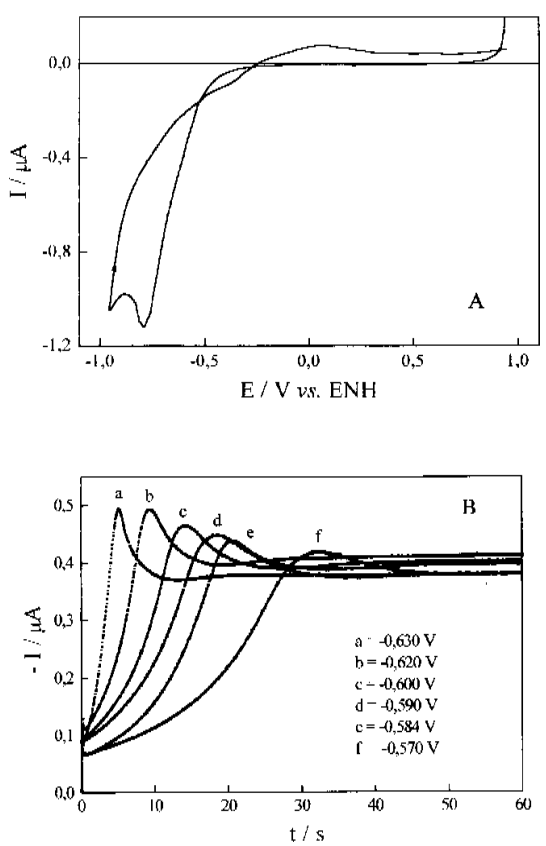

Figura 9. (A)Voltamograma cíclico para a deposição e redissolução de $\mathrm{Ni}$ sobre UME de Pt $(\phi=40 \mu \mathrm{m}) \mathrm{em} \mathrm{NiSO}_{4} 330 \mathrm{~g} \mathrm{~L}^{-1}+\mathrm{Ni}\left(\mathrm{NO}_{3}\right)_{2} 45 \mathrm{~g}$ $\mathrm{L}^{-1}+\mathrm{H}_{3} \mathrm{BO}_{3} 37 \mathrm{~g} \mathrm{~L}^{-1}$, a $0,1 \mathrm{Vs}^{-1}$ e (B) Transientes potenciostáticos para a nucleação de Ni sobre UME de Pt $(\phi=40 \mu \mathrm{m})$ no mesmo eletrólito.

núcleos mostrou-se controlado por transferência eletrônica, o modelo de Abyaneh ${ }^{38}$ desenvolvido para eletrodos convencionais revelou-se bastante satisfatório ${ }^{39}$.

\section{B. Eletroanalítica}

\section{i) Determinação conjunta de metais em água ${ }^{40}$}

Este foi um dos primeiros trabalhos a serem desenvolvidos no GMEME utilizando UME de fibra de carbono de $7 \mu \mathrm{m}$ de 
diâmetro. A crescente preocupação com a contaminação ambiental por metais pesados, como $\mathrm{Pb}, \mathrm{Hg}$ e $\mathrm{Cd}$, tem atraído a atenção de pesquisadores para o desenvolvimento de metodologias analíticas automatizadas para monitoramento ambiental destes poluentes. A utilização de técnicas eletroanalíticas com UME é uma das melhores alternativas para estas rotinas automatizadas. Neste trabalho, a voltametria de pulso diferencial com redissolução anódica foi utilizada para determinar $\mathrm{Cd}$ e $\mathrm{Pb}$ de maneira simultânea em matrizes sintéticas, feitas em laboratório, pelo acréscimo de diversas concentrações dos metais em tampão acetato. $\mathrm{O}$ eletrodo de fibra de carbono era recoberto com mercúrio, previamente depositado de uma solução de $\mathrm{Hg}\left(\mathrm{NO}_{3}\right)_{2}$ $5 \times 10^{-4} \mathrm{M}$. A menor concentração de chumbo adicionada ao tampão foi de $4,8 \times 10^{-7} \mathrm{M}$ e a de cádmio $8,9 \times 10^{-7} \mathrm{M}$.

$\mathrm{Na}$ figura 10 está representado um voltamograma de pulso diferencial executado a $5 \mathrm{mV} \mathrm{s}^{-1}$ com uma amplitude de $25 \mathrm{mV}$ pico a pico, mostrando os picos de redissolução dos metais depositados em $-1,1 \mathrm{~V}$ durante $60 \mathrm{~s}$, nas concentrações acima. A excelente definição dos picos voltamétricos indica a possibilidade de se utilizar este método para concentrações até dez vezes menores, atingindo um limite de detecção na ordem de $10 \mathrm{ppb}$. A utilização de UME tornou possível a obtenção de um limite de detecção razoavelmente baixo e possibilitou a execução do experimento em condições favoráveis em relação ao tempo do experimento e à preparação das amostras.

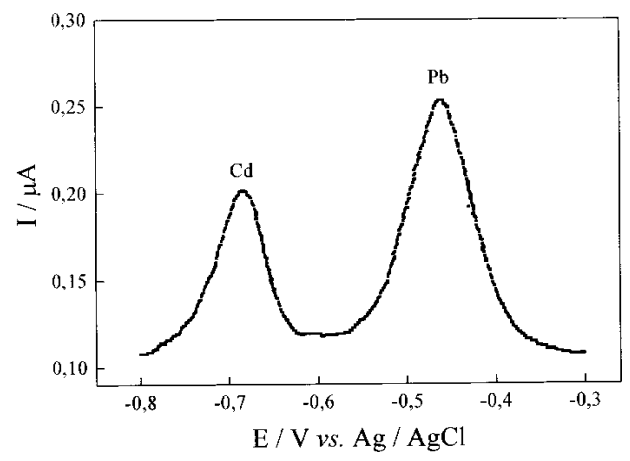

Figura 10. Voltamograma de redissolução anódica de pulso diferencial para a determinação simultânea de $\mathrm{Cd}$ e $\mathrm{Pb}$ em tampão acetato, usando UME de fibra de carbono $(\phi=7 \mu \mathrm{m}) .\left[\mathrm{Pb}^{2+}\right]=4,8 \times 10^{-8} \mathrm{M}$ e $\left[\mathrm{Cd}^{2+}\right]=3,1 \times 10^{-7} \mathrm{M}$. $E_{\text {dep }}=-1,2 \mathrm{~V} ; t_{\text {dep }}=600 \mathrm{~s}$, amplitude de pulso de $25 \mathrm{mV}$ pico a pico e $\mathrm{v}=1 \mathrm{~V} \mathrm{~s} \mathrm{~s}^{\mathrm{I}}$.

\section{ii) Determinação de nitritos em águas naturais ${ }^{41}$}

A presença de nitritos em água potável pode ocasionar graves danos à saúde, principalmente em crianças. $\mathrm{O}$ nitrito pode reagir com a hemoglobina no organismo, impedindo o transporte do oxigênio. Além disto, seus produtos metabólicos são, freqüentemente, cancerígenos. Com a utilização de nitritos em diversos produtos como conservantes para alimentos e remédios, torna-se cada dia mais importante o desenvolvimento de metodologias adequadas para a sua determinação. Os métodos tradicionalmente empregados são demorados e envolvem a utilização de substâncias tóxicas. Assim, UME de fibra de carbono de $7 \mu \mathrm{m}$ de diâmetro e de ouro de $15 \mu \mathrm{m}$ foram utilizados para a determinação direta de nitritos em águas naturais por voltametria de varredura linear.

$\mathrm{Na}$ figura 11 encontram-se voltamogramas de varredura linear para a oxidação de nitrito (a nitrato) em água mineral comercial não desaerada, sobre UME de fibra de carbono a $0,1 \mathrm{~V} \mathrm{~s}^{-1}$, após a subtração da curva do branco (mesma solução sem acréscimo de nitrito). As amostras de água mineral utilizadas não continham nitrito ou qualquer outra espécie interferente em quantidades detectáveis, pois o voltamograma do branco não apresentou qualquer onda na região de potenciais em estudo. Nesta mesma figura também se observa a curva de trabalho obtida representando as correntes limites de difusão, tomadas em 1,35 V em função das concentrações de nitrito adicionado. A excelente relação linear obtida permite calcular um limite de detecção (LD) de $8,8 \mu \mathrm{M}$ de acordo com a equação ${ }^{41}$ :

$$
\mathrm{LD}=3 \sigma / \mathrm{tg}
$$

onde 3 é um fator designado pela IUPAC, $\sigma$ o desvio padrão obtido da repetição das curvas do branco e tg a tangente da curva de calibração. Deve-se acrescentar que estas medidas foram efetuadas sem qualquer manipulação das amostras de água mineral. Assim, esta técnica utilizando UME se mostra extremamente vantajosa em relação ao tempo e custo das análises.

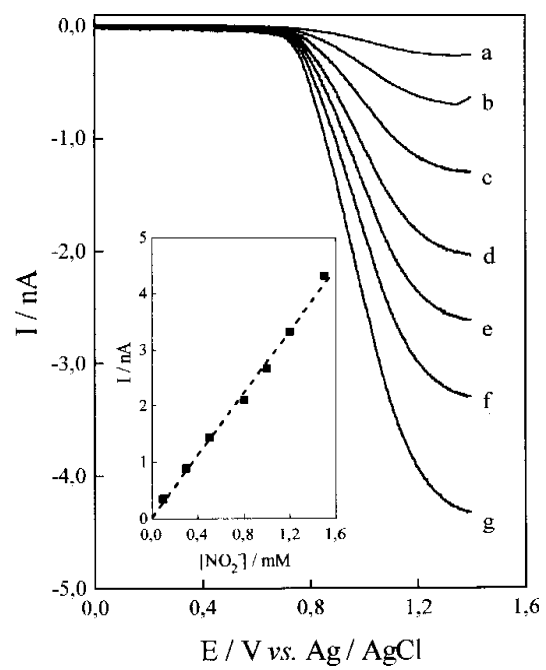

Figura 11. Voltamogramas de varredura linear, em amostra de água mineral sem desaeração, sobre UME de fibra de carbono $(\phi=7 \mu \mathrm{m})$, com diferentes concentrações de nitrito: (a) $1 \times 10^{-4}$, (b) $3 \times 10^{-4}$, (c) $5 \times 10^{-4},(d)$ $8 \times 10^{-4}$, (e) $1 \times 10^{-3}$, (f) $1,2 \times 10^{-3}$ e (g) $1,5 \times 10^{-3} \mathrm{M} . v=0,1 \mathrm{~V} \mathrm{~s}^{-1}$. A figura inserida representa a curva analítica, obtendo-se as correntes em 1,35 V.

\section{iii) Determinação de oxigênio em águas naturais ${ }^{42}$}

A determinação do teor de oxigênio em águas naturais, poluídas ou não, em rios ou lagos, tem sido importante no sentido da recuperação de mananciais poluídos, assim como em estações de tratamento de esgotos e em grandes lagos artificiais vinculados a hidroelétricas. Os métodos químicos tradicionais de determinação de oxigênio são bastante trabalhosos, como o método de Winkler ${ }^{43,44}$, que possui várias modificações para levar em consideração os diversos interferentes existentes. Estes métodos exigem uma grande manipulação da amostra e são sujeitos a muitas interferências não compensadas. Para aumentar a rapidez e diminuir os custos das análises, foram desenvolvidos sensores de oxigênio, do tipo Clark $^{45}$, para a utilização direta na amostra, sem tratamento químico. Estes sensores são constituídos de eletrodos de $\mathrm{Pt}$, que fazem uma medida da corrente de redução do oxigênio a um dado potencial. Entretanto, estes sensores, embora bastante seletivos, ainda são lentos, demorando vários minutos para estabilizar e são sujeitos a alguns interferentes que podem obstruir a membrana utilizada para conter o eletrólito. Como uma alternativa, neste trabalho, UME de $\mathrm{Pt}(\phi=40 \mu \mathrm{m})$ e $\mathrm{Au}(\phi=25 \mu \mathrm{m})$ foram utilizados para a determinação direta do $\mathrm{O}_{2}$ dissolvido por voltametria cíclica. As principais vantagens encontradas com a utilização desta metodologia foram: (a) devido ao tamanho reduzido dos UME, é possível aplicá-los em condições onde seja necessário não perturbar o meio em estudo; (b) a técnica voltamétrica utilizada permite efetuar um condicionamento 
superficial do eletrodo pela varredura consecutiva em um dado intervalo de potenciais, eliminando a interferência de contaminantes e (c) o efeito de impurezas adsorvidas na superfície do eletrodo é facilmente detectável na resposta do branco (obtido nas mesmas condições do experimento, porém sem a presença do $\mathrm{O}_{2}$ ). A figura 12 apresenta os resultados obtidos sobre $\mathrm{Pt}$ para uma solução de $\mathrm{H}_{2} \mathrm{SO}_{4} 0,1 \mathrm{M}$, com acréscimo de diferentes concentrações de oxigênio. $\mathrm{O}$ deslocamento do perfil voltamétrico da Pt serve para se obter a curva de polarização para a redução do $\mathrm{O}_{2}$. As correntes limites de difusão obtidas após a subtração do branco, foram representadas em função do teor de $\mathrm{O}_{2}$ na reta de trabalho apresentada no "insert" da figura 12 . As concentrações de $\mathrm{O}_{2}$ da reta de trabalho foram determinadas pelo método de Winkler. Esta reta pode então ser utilizada para futuras determinações em amostras de $\mathrm{H}_{2} \mathrm{SO}_{4}$ de conteúdo desconhecido de $\mathrm{O}_{2}$. O mesmo procedimento foi aplicado com sucesso para UME de Au. Este método foi ainda testado para amostras naturais, como águas de rios poluídos ou não, mostrando uma boa tolerância a contaminantes orgânicos (como ácidos húmicos e fúlvicos e fenóis), como pode ser observado nas curvas de trabalho apresentadas na figura 13 para Pt lisa, Pt recoberta com Nafion ${ }^{\circledR}$ e Au liso, todos em amostras de água de rio urbano. Nesta figura, pode-se observar que o UME de Pt recoberto com Nafion ${ }^{\circledR}$ e o de Au apresentam maior sensibilidade que o de $\mathrm{Pt}$ liso. Isto deve ser atribuído à grande facilidade de espécies contaminantes se adsorverem na superfície da Pt lisa. O recobrimento com Nafion ${ }^{\circledR}$, assim como a utilização da superfície de $\mathrm{Au}$, minimiza este efeito, com o aumento da sensibilidade do eletrodo.

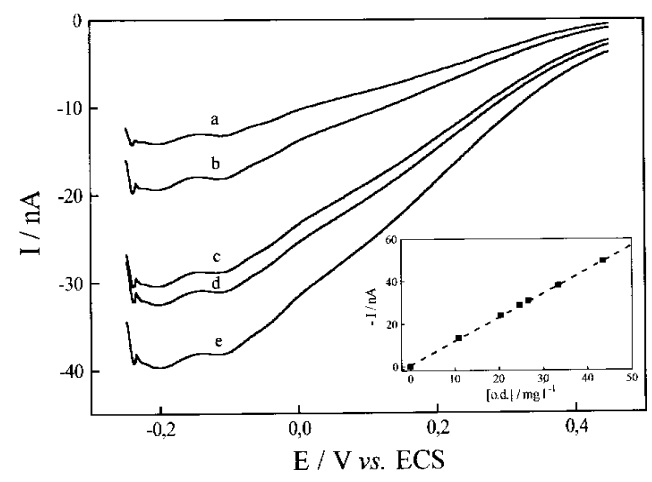

Figura 12. Curvas voltamétricas (após subtração do branco), para a redução de $\mathrm{O}_{2}$ sobre $\mathrm{UME}$ de Pt $(\phi=40 \mu \mathrm{m})$, em meio de $\mathrm{H}_{2} \mathrm{SO}_{4} 0,1 \mathrm{M}$ saturado com várias concentrações de oxigênio: (a) 10, (b) 20, (c) 25, (d) 27 e (e) $32 \mathrm{mg} \mathrm{L}^{-1}$. v $=0,2 \mathrm{~V} \mathrm{~s}^{-1}$. A figura inserida representa a curva de trabalho obtida pelas correntes a $-0,2 \mathrm{~V}$.

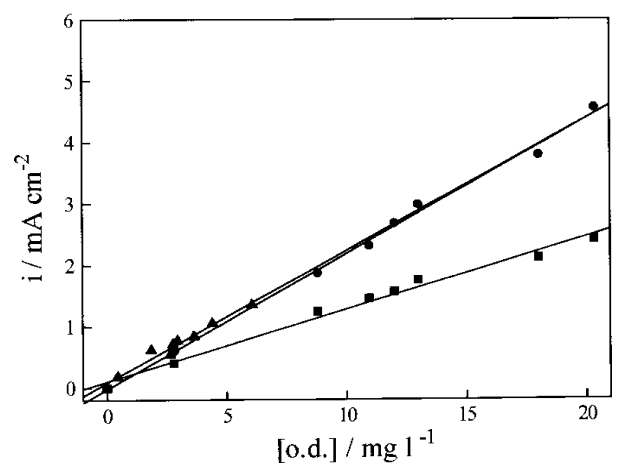

Figura 13. Curvas de calibração para amostras de água de rio urbano: (•) UME de Pt lisa $(\phi=40 \mu \mathrm{m})$, saturação com $\mathrm{O}_{2}$ puro; (•) UME de Pt $(\phi=40 \mu \mathrm{m})$ recoberta com Nafion ${ }^{\circledR}$, saturação com $\mathrm{O}_{2}$ puro e (₫) UME de $\mathrm{Au}(\phi=25 \mu \mathrm{m})$, saturação com $\mathrm{O}_{2}$ existente no ar sintético.

\section{iv) Determinação de metais pesados por potenciometria de redissoluçã̃o 46,47}

A análise potenciométrica por redissolução é uma técnica que se baseia na redução potenciostática e amalgamação de íons metálicos, seguida por oxidação química. A análise consiste, assim, de duas fases: uma etapa de deposição (eletroquímica) e uma de redissolução (química). Durante a aplicação do potencial de redução (eletrólise) os metais são depositados em um eletrodo recoberto por um filme de mercúrio. Os íons são concentrados no amálgama a um nível muito maior que na solução, permitindo limites de detecção extremamente baixos. A seguir, o potencial de deposição é removido e nenhuma corrente passa pelo eletrodo. Este começa, então, a descarregar e o potencial atinge o valor do potencial redox do agente oxidante acrescentado para redissolver os metais amalgamados (por exemplo $\mathrm{Hg}(\mathrm{II})$ ). A medida do potencial do eletrodo em função do tempo fornece informações qualitativa e quantitativa dos metais presentes, de acordo com:

$$
\tau=\mathrm{k}\left[\mathrm{M}^{\mathrm{n}+}\right] \mathrm{t}_{\mathrm{d}}
$$

onde $\tau$ é o sinal analítico equivalente ao tempo de redissolução, $\mathrm{k}$ é uma constante, $\left[\mathrm{M}^{\mathrm{n}+}\right]$ a concentração do íon metálico em solução e $t_{d}$ o tempo de deposição. A identificação qualitativa dos diferentes elementos determinados por análise potenciométrica por redissolução pode ser feita baseada nos potenciais padrões de cada metal dado pela lei de Nernst. A análise quantitativa é feita pelo método da adição de padrão na matriz em estudo.

Desenvolveu-se uma instrumentação consistindo de uma interface AD/DA em um computador IBM-PC 48666 MHz com um "software" apropriado e um sistema de chaveamento para a interrupção da corrente aplicada na primeira etapa, ligando um UME de $\mathrm{Pt}$ recoberto com $\mathrm{Hg}$ e um eletrodo de referência miniaturizado de $\mathrm{Ag} / \mathrm{AgCl}$, para análises potenciométricas.

A metodologia para determinação de alguns metais em vinagre e líquidos de conserva ${ }^{48}$ está sendo desenvolvida sem a necessidade de decomposição prévia da matéria orgânica nem desoxigenação da amostra. Isto reduz significativamente o tempo de análise e elimina os riscos de contaminação na adição de reagentes. As figuras 14 e 15 mostram os potenciogramas típicos para a determinação de $\mathrm{Zn}^{2+}$ em amostra de líquido de conserva de palmito in natura e $\mathrm{Pb}^{2+}$ em amostra de líquido de conserva de cogumelo com um UME de Pt de $10 \mu \mathrm{m}$ recoberto com $\mathrm{Hg}$, juntamente com as respectivas curvas analíticas. Esta técnica apresenta inúmeras vantagens que estão discutidas em um trabalho submetido a esta revista ${ }^{49}$.

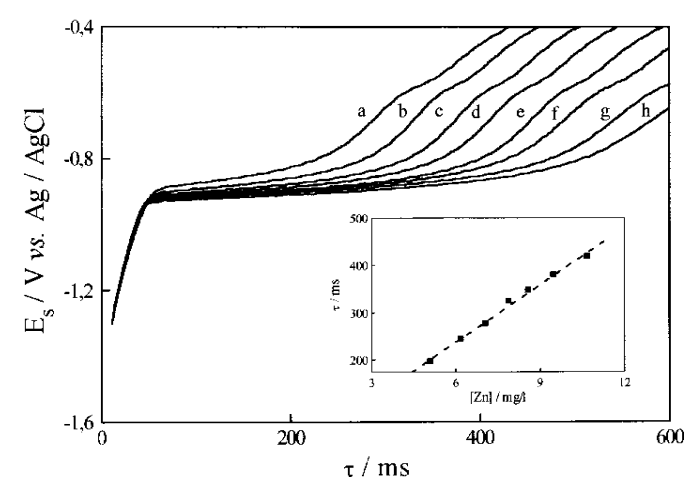

Figura 14. Potenciogramas de redissolução para determinação de $\mathrm{Zn}^{2+}$, em várias concentrações: (a) 5,09, (b) 6,17, (c) 7,05, (d) 7,89, (e) 8,60, (f) 9,47, (g) 10,70 e (h) 12,90 $\mathrm{mg} \mathrm{L}^{-1}$, em amostra de líquido de conserva de palmito in natura. $\left[\mathrm{Hg}^{2+}\right]=1,2 \times 10^{-4} \mathrm{M} ; E_{\text {dep }}=-1,45$ $V ; t_{\text {dep }}=45 \mathrm{~s}$. Inserido, tem-se as linearidades entre $\tau$ e $\left[\mathrm{Zn}^{2+}\right]$. 


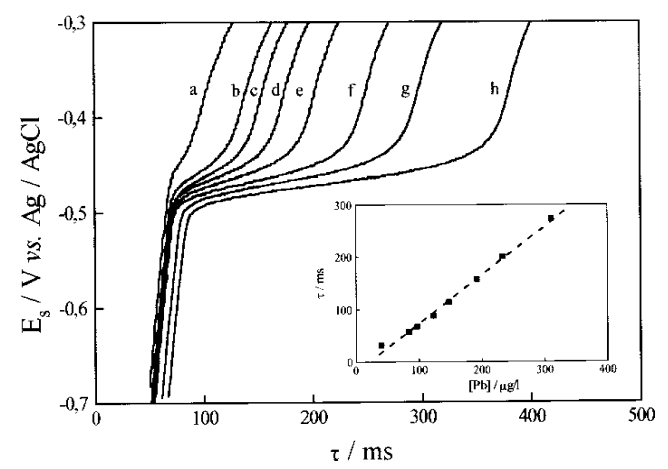

Figura 15. Potenciogramas de redissolução para determinação de $\mathrm{Pb}^{2+}$, em várias concentrações: (a) 40,9; (b) 83,8; (c) 97,2; (d) 123; (e) 148; (f) 193; (g) 234 e (h) $311 \mu g L^{-1}$, em amostra de conserva de cogumelo diluída $70 \%$. $\left[\mathrm{Hg}^{2+}\right]=6,1 \times 10^{-4} \mathrm{M} ; E_{\text {dep }}=-1,35 \mathrm{~V} ; t_{\text {dep }}=$ $300 \mathrm{~s}$. Inserido, tem-se as linearidades entre $\tau e\left[\mathrm{~Pb}^{2+}\right]$.

\section{CONCLUSÕES}

Os exemplos discutidos mostram uma ampla faixa de aplicações de UME em eletroquímica e eletroanalítica. Sob diversos pontos de vista, estes sistemas têm introduzido uma abordagem inteiramente nova para problemas que não apresentavam soluções com técnicas tradicionais.

Os UME propiciam a especial condição de miniaturização da instrumentação eletroquímica necessária. Entretanto, devido ao elevado preço de UME comerciais, é extremamente conveniente construí-los no próprio laboratório. Neste trabalho, foi mostrada uma alternativa de construção de UME que tem sido utilizada com sucesso há, pelo menos, dez anos.

A grande vantagem da utilização destes UME em eletroquímica está relacionada com a possibilidade de se minimizar os problemas com interferentes nas respostas obtidas. Assim, não se observam efeitos de queda ôhmica, bolhas, correntes capacitivas, etc.

No campo da eletroanalítica, os UME possibilitam a diminuição dos limites de detecção nas técnicas voltamétricas, eliminam a necessidade de utilização de eletrólito de suporte em análises e proporcionam respostas com menos ruídos e com maior reprodutibilidade.

Desta forma, o futuro dos UME em eletroquímica e eletroanalítica parece apontar para um aumento exponencial de aplicações nos mais diferentes sistemas.

\section{AGRADECIMENTOS}

Os autores agradecem a Fernando Crnkovic pela elaboração dos desenhos e ao CNPq, CAPES e FINEP pelos auxílios concedidos.

\section{REFERÊNCIAS}

1. Seddon, B. J.; Shao, Y.; Girault, H. H.; Electrochim. Acta 1994, 39, 2377.

2. Pletcher, D.; Valdes, E. M.; Anal. Chim. Acta 1991, 246, 267.

3. Kuhr, W. G.; Barrett, V. L.; Gagnon, M. R.; Hopper, P.; Pantano, P.; Anal. Chem. 1993, 65, 617.

4. Malinski, T.; Taha, Z.; Grunfeld, S.; Burewicz, A.; Tomboulian, P.; Kiechle, F.; Anal. Chim. Acta 1993, 279, 135.

5. Wang, J.; Sucman, E.; Tian, B.; Anal. Chim. Acta 1994, 286, 189.

6. Motonaka, J.; Kamizasa, M.; Faulkner, L. R.; J. Electroanal. Chem. 1994, 373, 75.

7. Wang, J.; Lu, J.; Tian, B.; Yarnitzky, C.; J. Electroanal. Chem. 1993, 361, 77.

8. Baldo, M. A.; Daniele, S.; Mazzocchin, G. A.; Anal. Chim. Acta 1993, 272, 151.
9. Daniele, S.; Baldo, M. A.; Ugo, P.; Mazzocchin, G. A.; Anal. Chim. Acta 1990, $238,357$.

10. Tavares, M. C.; Machado, S. A. S.; Mazo, L. H.; Avaca, L. A., In: Anais, $43^{\text {rd }}$ ISE Meeting, Cordoba, Argentina, 20-25 de Setembro, 1992, p. 523.

11. Daniele, S.; Lavagnini, I.; Baldo, M. A.; Magno, F.; $J$. Electroanal. Chem. 1996, 404, 105.

12. Howell, J. O.; Wightman, R. M.; Anal. Chem. 1984, 56, 524.

13. Szulborska, A.; Baranski, A.; J. Electroanal. Chem. 1994, 377, 269.

14. Alden, J. A.; Compton, R. G.; Dryfe, R. A. W.; J. Electroanal. Chem. 1995, 397, 11.

15. Compton, R. G.; Wellington, R. G.; Dobson, P. J.; Leigh, P. A.; J. Electroanal. Chem. 1994, 370, 129.

16. Hills, G.; Pour, A. K.; Scharifker, B.; Electrochim. Acta 1983, 28, 891.

17. Peña, M. J.; Celdran, R.; Duo, R.; J. Electroanal. Chem. 1994, 367, 85.

18. Cooper, J. B.; Bond, A. M.; Anal. Chem. 1993, 65, 2724.

19. Daniele, S.; Mazzocchin, G. A.; Anal. Chim. Acta 1993, $273,3$.

20. Fleischmann, M.; Pons, S.; Rolison, D. R.; Schmidt, P. P. (eds.), Ultramicroelectrodes, Datatech, Morgaton, North Carolina, 1987.

21. Wightman, R. M.; Wipf, D. O.; em A. J. Bard (ed.) Electroanalytical Chemistry, vol. 15, Marcel Dekker, New York, 1989, p. 267.

22. Montenegro, M. I.; Queirós, M. A.; Daschbach, J. L. (eds.), Microelectrodes: Theory and Applications, Kluwer Academic Publishers, The Netherlands, 1991, p. 497.

23. Scharifker, B. R.; em J. O' M. Bockris, B. E. Conway e R. E. White (eds.) Modern Aspects of Electrochemistry, $\mathrm{n}^{\mathrm{o}} 22$, Plenum Press, New York, 1992, p. 467.

24. a) Montenegro, M. I.; Portugaliae Electrochim. Acta 1985, 3, 165; b) Montenegro, M. I.; em R. G. Compton e G. Hancock (eds.) Research in Chemical Kinetics, vol. 2, Elsevier, The Netherlands, 1994, p. 1.

25. Correia, A. N.; Mascaro, L. H.; Machado, S. A. S.; Mazo, L. H.; Avaca, L. A.; Quím. Nova 1995, 18, 475.

26. Bond, A. M.; Fleischmann, M.; Robinson, J.; J. Electroanal. Chem. 1984, 168, 299.

27. Scharifker, B.; Hills, G.; J. Electroanal. Chem. 1981, $130,81$.

28. Whelan, K. P.; O’Dea, J. J.; Osteryoung, J.; Aoki, K.; J. Electroanal. Chem. 1986, 202, 23.

29. Wehmeyer, K. R.; Deakin, M. R.; Wightman, R. M.; Anal. Chem. 1985, 57, 1913.

30. Beden, B.; Kadirgan, F.; Lamy,C.; Leger, J. M.; J. Electroanal. Chem. 1982, 142, 171.

31. Woods, R.; Em: A. J. Bard (ed.) Advances in Electroanalytical Chemistry, vol. 9, Marcel Dekker, New York, 1976, p. 1.

32. Machado, S. A. S.; Mazo, L. H.; Avaca, L. A.; Inst. Sci. Tech. 1994, 22, 61 .

33. Correia, A. N.; Machado, S. A. S.; Electrochim. Acta 1997, no prelo.

34. Southampton Electrochemistry Group, Instrumental Methods in Electrochemistry, cap.9, Ellis Horwood, John Wiley \& Sons, England, 1985, p. 283.

35. Scharifker, B.; Hills, G.; Electrochim. Acta 1983, $28,879$.

36. Aoki, K.; Osteryoung, J.; J. Electroanal. Chem. 1984, $160,335$.

37. Correia, A. N.; Machado, S. A. S.; Avaca, L. A.; J. Braz. Chem. Soc. 1994, 5, 173.

38. Abyaneh, M. Y.; J. Electroanal. Chem. 1986, 209, 1.

39. Correia, A. N.; Machado, S. A. S.; J. Braz. Chem. Soc. 1997, 8, 71.

40. Machado, S. A. S.; Alves, C. R.; Mazo, L. H. e Avaca, L. A.; 15a Reunião Anual da SBQ, Caxambú - Minas Gerais, 27 a 31 de Maio, 1992, resumo QA-044. 
41. Silva, S. M.; Alves, C. R.; Machado, S. A. S.; Mazo, L. H.; Avaca, L. A.; Electroanalysis 1996, 8, 1055.

42. Martins, M. R.; Tese de doutorado, IQSC-USP, 1996.

43. Golterman, H. L.; Clymo, R. S.; Ohnstad, M. A. M; Methods for Physical and Chemical Analysis of Fresh Waters, Blackwell Scientific Publications, 2a ed., 1978.

44. Standard Methods for Examination of Water and Waste-
Water, $16^{\text {th }}$ ed., American Public Health Association, USA, AWWA WPCF; 1995.

45. Clark, L.; Lyons, C.; Am. N. Y. Acad. Sci. 1962, 102, 29. 46. Jagner, D.; Graneli, A.; Anal. Chim. Acta 1976, 83, 19. 47. Nobre, A. L. R.; Tese de doutorado, IQSC-USP, 1996. 48. Hussam, A.; Coetzee, J. F.; Anal. Chem. 1985, 57, 581. 49. Nobre, A. L. R.; Mazo, L. H.; Quím. Nova 1996, $20,412$. 